Proyecciones

Vol. 20, No 1, pp. 93-126, May 2001.

Universidad Católica del Norte

Antofagasta - Chile

\title{
SCHOTTKY UNIFORMIZATIONS AND RIEMANN MATRICES OF MAXIMAL SYMMETRIC RIEMANN SURFACES OF GENUS 5*
}

\author{
RUBÉN HIDALGO \\ Universidad Técnica Federico Santa María-Chile
}

\begin{abstract}
In this note we consider pairs $(S, \tau)$, where $S$ is a closed Riemann surface of genus five and $\tau: S \rightarrow S$ is some anticonformal involution with fixed points so that $K(S, \tau)=\{h \in$ Aut $\left._{ \pm}(S): h \tau=\tau h\right\}$ has the maximal order 96 and $S / \tau$ is orientable. We observe that there are exactly two topologically different choices for $\tau$. They give non-isomorphic groups $K(S, \tau)$, each one acting topologically rigid on the respective surface $S$. These two cases give then two (connect) real algebraic sets of real dimension one in the moduli space of genus 5 . In this note we describe these components by classical Schottky groups and with the help of these uniformizations we compute their Riemann matrices.
\end{abstract}

Keywords : Schottky groups, Riemann surfaces, Riemann matrices.

Subjclass : Primary 30F40.

*Partially supported by projects UTFSM 12.01.22, Fondecyt 1000715 and a Presidential Chair on Geometry 


\section{Introduction}

A closed Riemann surfaces $S$ of genus $g \geq 2$ is called symmetric if it admits an anticonformal involution $\tau: S \rightarrow S$, called a symmetry of $S$. If the symmetry $\tau$ has fixed points, then we refer to it as a reflection. We denote by $K(S, \tau)$ the total group of conformal/anticonformal automorphisms of $S$ commuting with $\tau$ and by $K^{+}(S, \tau)$ its index two subgroup of conformal automorphisms. The quotient $R=S / \tau$ is a compact (possible bordered) Klein surface of algebraic genus $g$. The number of possible border components is equal to number of fixed point components of $\tau$. The group $H(S, \tau)=K(S, \tau) / \tau$ is isomorphic to the full group of automorphisms of $R$. In the case $\tau$ is a reflection, we have that $S / \tau$ has non-empty boundary and, as consequence of [18] and [19], we have that the order of $K(S, \tau)$ is at most $24(g-1)$. We say that $(S, \tau)$ is maximal symmetric if the maximal order $24(g-1)$ is attained for $K(S, \tau)$; the surface $S$ is then said to be maximal symmetric and the reflection $\tau$ to be a maximal reflection (we must not get confused with the definition of a M-curve which means $\tau$ to be a reflection with the maximal number of components of fixed points). It is also important to note that the bound $24(g-1)$ is attained by an infinite number of values of $g$ and also it is not attained for an infinite values of $g$. It is a well known fact that any closed symmetric Riemann surface $S$ with a reflection $\tau: S \rightarrow S$ can be uniformized by a classical Schottky group [7], [16], [21] and [24]. In fact, such a Schottky group can be chosen to keep invariant a circle $C$ so that the reflection on $C$ is a lifting of $\tau$ [16] and, in particular, $K(S, \tau)$ lifts to such an Schottky uniformization of $S$ (see section 2). This type of Schottky uniformizations and Burnside's arguments [2], which we recall in section 4 , we may be able to compute the respective Riemann matrices of the surface $S$. In the case that $(S, \tau)$ is maximal symmetric we know that $S / K^{+}(S, \tau)$ is the Riemann sphere branched at four values with orders 2, 2, 2 and 3, respectively [12]. The reflection $\tau: S \rightarrow S$ induces a reflection of the sphere $S / K^{+}(S, \tau)$ permuting these branch values and, in particular, it follows that $S / K(S, \tau)$ is either: (i) a closed disc with four branched values, of orders $2,2,2$ and 3 , on its border or (ii) a closed disc with one branch value in its interior, of order 2 , and two branched values on its border of orders 2 and 3 . Using the fact that 
the NEC group uniformizing $S / K(S, \tau)$ contains a bordered surface group $F$, uniformizing $S / \tau$, as normal subgroup, we have that case (ii) is not possible. As a consequence, for a maximal symmetric Riemann surface $S$ with a maximal reflection $\tau: S \rightarrow S$ we must have that $S / K(S, \tau)$ is a closed disc with exactly four branched values of orders $2,2,2$ and 3 on its border.

In this note we consider maximal symmetric Riemann surfaces $S$ of genus five with a maximal reflection $\tau: S \rightarrow S$ for which $S / \tau$ is orientable, that is, the set of fixed points of $\tau$ disconect $S$ into two surfaces. In section 5 we observe that there are exactly two different topological action of $K(S, \tau)$. Each one of these topological actions describes a real one dimensional locus in moduli space. In each of these two cases, there is a normal subgroup $H \cong \mathcal{Z}_{2}+\mathcal{Z}_{2}$ of $K(S, \tau)$, containing only conformal automorphisms and acting freely. The quotient $S / H$ is a closed Riemann surface of genus two admitting the group $K(S, \tau) / H$ as group of automorphisms. This group is isomorphic to $\mathcal{Z}_{2}+\mathcal{Z}_{2}+D_{3}$, where $D_{3}$ is a conformal group isomorphic to the dihedral group of order 6 , one of the $\mathcal{Z}_{2}$ is generated by the hyperelliptic involution and the other $\mathcal{Z}_{2}$ is generated by the reflection induced by $\tau$. We obtain in this way the exactly two possibilities for such an action in genus two. In section 6 we describe a particular uniformization of the closed unit disc with four branch values of orders 2, 2, 2 and 3 on its border. This uniformization is given by a Kleinian group $\widehat{K}$, generated by four reflections, having a connected region of discontinuity. In section 7 we describe two Schottky normal subgroup of genus two $G_{2}$ and $F_{2}$ of index 24 in $\widehat{K}$. The Schottky groups $G_{2}$ and $F_{2}$ uniformize the closed Riemann surfaces of genus two obtained in section 3 . We also describe an algebraic limit giving a noded Schottky group. With the help of these Schottky uniformizations and results due to W. Burnside [2] we compute the Riemann matrices of these surfaces. In section 8 we proceed to find a Schottky normal subgroup $G_{5}$ (respectively, $F_{5}$ ) of the Schottky group $G_{2}$ (respectively, $F_{2}$ ) of index 4 , which is also normal in the group $\widehat{K}$. These groups uniformize all the Riemann surfaces of genus 5 described in section 5 . We also describe some algebraic limits giving a couple of noded Schottky groups. These noded Schottky groups then uniformize boundary points in Moduli space of the above Riemann surfaces with automorphism. We proceed again 
to use these Schottky uniformizations and results due to W. Burnside [2] to compute the Riemann matrices of these surfaces.

\section{Schottky Uniformizations}

Let $g$ be some positive integer. Assume we have a collection of $2 g$ pairwise disjoint simple loops, say $\alpha_{1}, \ldots, \alpha_{g}, \widetilde{\alpha}_{1}, \ldots, \widetilde{\alpha}_{g}$, bounding a common region $\mathcal{D}$ of connectivity $2 g$. Let us also assume we have a collection of Moebius transformations $A_{1}, \ldots, A_{g}$ (necessarily loxodromic ones) so that $A_{j}\left(\alpha_{j}\right)=\widetilde{\alpha}_{j}$ and $A_{j}(\mathcal{D}) \cap \mathcal{D}=\emptyset$, for all $j=1, \ldots, g$. The group $J$ generated by $A_{1}, \ldots, A_{g}$ turns out to be a Kleinian group, purely loxodromic, isomorphic to a free group of rank $g$ and with connected region of discontinuity [15], called a Schottky group of genus $g$ (for another equivalent definitions of Schottky groups one can check [17] and [5]). The domain $\mathcal{D}$ is called a standard fundamental domain for $J$, the loops $\alpha_{1}, \ldots, \alpha_{g}, \widetilde{\alpha}_{1}, \ldots, \widetilde{\alpha}_{g}$ are called a fundamental set of loops.

For a Schottky group $J, \Omega / J$ is a closed Riemann surface of genus $g$, where $\Omega$ denotes the region of discontinuity of $J$. The classical retrosection theorem states that if $S$ is a closed Riemann surface of genus $g$, then there is a Schottky group $J$ with region of discontinuity $\Omega$ and $P: \Omega \rightarrow S$ a normal covering map, with $J$ as covering group. We say that $(J, \Omega, P: \Omega \rightarrow X)$ is a Schottky uniformization of $S$. A simple proof of this fact was also given by L. Bers in [1] using quasiconformal mappings.

A Schottky group $J$ is called hyperelliptic if there is a Moebius transformation $C$ of order two such that $C J C^{-1}=J$ and the group generated by $J$ and $C$ uniformizes an sphere (necessarily with $2(g+1)$ branched points of order two). Equivalently, there is a set of free generators $A_{1}, \ldots, A_{g}$ for $G$ so that $C A_{j} C=A_{j}^{-1}$ for $j=1, \ldots, g$. The hyperelliptic Schottky groups uniformize hyperelliptic Riemann surfaces and, reciprocally, every hyperelliptic Riemann surface can be uniformized by such a group [13].

In the case we can choose the above loops $\alpha_{j}$ and $\widetilde{\alpha}_{j}$ (for all $j$ ) as Euclidean circles, for some set of free generators of the Schottky group, we say that $J$ is a classical Schottky group. The existence of non-classical Schottky groups is well known [14]. An open problem is to determine if every closed Riemann surface can be uniformized by 
a classical Schottky group. Related to this problem, B. Maskit has proved the following:

Theorem 2.1 [16]. Let $S$ be a closed Riemann surface admitting a reflection $\tau: S \rightarrow S$. Then there is a classical Schottky group preserving an Euclidean circle $\mathcal{C}$ on the Riemann sphere, that is, either is a Fuchsian group or is an extended Fuchsian group (it is not Fuchsian but contains an index two subgroup which is Fuchsian) uniformizing $S$. The reflection on $\mathcal{C}$ is a lifting of the reflection $\tau$.

In the case the reflection $\tau$ has the maximal number of components of fixed points, the proof of Maskit's result is simple. In fact, set $R=$ $S / \tau$ and $P: S \rightarrow R$ the anti-holomorphic branched covering induced by the action of $\tau$. The surface $R$ is an orientable closed surface of genus $p$ with $l$ boundary components, where $g+1=2 p+l$ and $l$ denotes the number of connected components of fixed points of $\tau$. Let $X$ be the interior of $R$. The set of fixed points of $\tau$ disconnect $S$ into two surfaces (open surfaces), say $S_{1}$ and $S_{2}$, with common boundary (the set of fixed points of $\tau$ ). The restriction of $P$ to $S_{1}$ produces a conformal homeomorphism between $X$ and $S_{1}$. Let us uniformize $X$ by a Fuchsian group $F$ acting on the unit disc $\Delta$, that is, there is a conformal covering $Q: \Delta \rightarrow X=\Delta / F$. The group $F$ is a Fuchsian group of the second kind and, in particular, a classical Schottky group of genus $g$. If we denote by $\Omega$ the region of discontinuity of $F$, then $\widehat{S}=\Omega / F$ is a closed Riemann surface of genus $g$ admitting a symmetry $\eta: \widehat{S} \rightarrow \widehat{S}$ so that $S / \tau=\widehat{S} / \eta$. Set $\widehat{S}_{1}=Q(\Delta)$ and $\widehat{S}_{2}=Q(\Omega-\bar{\Delta})$. If we denote by $L: \widehat{S} \rightarrow S / \tau$ the anticonformal branched covering induced by the action of $\eta$ on $\widehat{S}$, then the map $T: S \rightarrow \widehat{S}$ defined by $\left.T\right|_{\overline{S_{1}}}=\left.\left.L\right|_{\widehat{S}_{1}} ^{-1} P\right|_{S_{1}}$ and $\left.T\right|_{S_{2}}=\left.\left.L\right|_{\widehat{S}_{2}} ^{-1} P\right|_{S_{2}}$ turns out to be a biholomorphic map.

A lifting problem concerning Schottky uniformizations of closed Riemann surfaces is the following. Assume we have a closed Riemann surface $S$ and a group $H$ of conformal/anticonformal automorphisms of it. In general, there is no Schottky uniformization of $S$ for which the group $H$ lifts. It is then natural to ask for conditions in order to ensure the existence of a Schottky uniformization of $S$ for which this lifting is possible. When this is the case, we say that $H$ is of Schottky type. 
In [8] we have found easy necessary conditions to be satisfied by $H$, a group of conformal automorphisms, in order for it to be of Schottky type (condition $(\mathrm{A})$ ). These conditions are trivially satisfied by groups isomorphic to a dihedral group and for groups acting free fixed points. In [8], [9], [10] and [11] we have shown that these necessary conditions turn out to be sufficient for Abelian groups, dihedral groups, the alternating groups $\mathcal{A}_{4}$ and $\mathcal{A}_{5}$ and for the symmetric group $\mathcal{S}_{4}$. In the case of groups admitting orientation-reversing transformations, we have some results for the cyclic case in [4]. Maskit's result asserts the following

Corollary 2.2. Let $S$ be a closed Riemann surface with a reflection $\tau$. Then $K(S, \tau)$ is of Schottky type.

The reason of this is the following. Let us assume we have a closed Riemann surface $S$ and a reflection $\tau: S \rightarrow S$. Let $G$ be a Schottky group uniformizing $S$ so that the unit circle $S^{1}$ is invariant under $G$ and $\sigma$, the reflection on $S^{1}$, is a lifting of $\tau$ (as consequence of the above Maskit's result). Let us denote by $\Delta$ the unit disc. Set $\Omega$ the region of discontinuity of $G$ and denote by $P: \Omega \rightarrow S$ the holomorphic covering induced by $G$.

(i) Assume that the fixed points of $\tau$ divide $S$ into two surfaces, say $S_{1}$ and $S_{2}$. In this case, we have that $G$ is a fuchsian group. We may assume $S_{1}=P(\Delta)$. Set $K$ the index two subgroup of $K(S, \tau)$ that keeps $S_{1}$ invariant. We have then that $K(S, \tau)$ is generated by $K$ and $\tau$. Since $P: \Delta \rightarrow S_{1}$ is a universal covering, we can lift $K$. Such a lifting is a fuchsian group $\widehat{K}$. It is now clear that the group generated by $\sigma$ and $\widehat{K}$ is the lifting of $K(S, \tau)$.

(ii) Assume that the fixed points of $\tau$ do not divide $S$. Set as $\widehat{G}$ the group generated by $G$ and $\sigma$. Since the unit circle $S^{1}$ is invariant under $G$, we have that the order two subgroup $F$ of $\widehat{G}$ generated by $\sigma$ is a normal subgroup. We consider the branched covering $Q: \Omega \rightarrow \bar{\Delta}$ induced by the action of $F$. The quotient group $H=\widehat{G} / F$ acts as group of conformal automorphisms of the unit disc $\Delta$. We have that the holomorphic covering $Q: \Delta \rightarrow(S / \tau)^{*}$, induced by $H$ is a universal covering, where $(S / \tau)^{*}=(S-\operatorname{Fix}(\tau)) / \tau$. In particular, we can lift 
$K(S, \tau) / \tau$ to $\Delta$ by this universal covering. Such a lifting is given by Moebius transformations keeping invariant the unit disc and they extend in natural manner to act on the unit circle boundary. Now, it is easy to see how to lift any of these automorphisms of $\bar{\Delta}$ by the (branched) covering $Q$ to obtain in this way the lifting of $K(S, \tau)$ as desired.

In sections 6,7 , and 8 we deal with the explicit construction of classical Schottky groups of genus $g=5$ which uniformize maximal symmetric Riemann surfaces $S$ admitting a maximal reflection $\tau$ for which $S / \tau$ is orientable and $S / K(S, \tau)$ is a closed disc with branch values of orders 2, 2, 2 and 3 on its border. These Schottky groups have the property that they uniformize all the surfaces with such conditions.

\section{Riemann Matrices}

Let us assume we have a closed Riemann surface $S$ of genus $g$ together a group $K$ of automorphisms of it (maybe containing orientation reversing ones).

A symplectic basis of $S$ is a basis of the first homology group of $S$, with integer coefficients, whose intersection matrix is

$$
J=\left(\begin{array}{ll}
0 & I \\
-I & 0
\end{array}\right)
$$

where $I$ denotes the identity matrix of size $g \times g$.

To each symplectic basis of $S$, say $\left\{\alpha_{1}, \ldots, \alpha_{g}, \beta_{1}, \ldots, \beta_{g}\right\}$, we have associated a dual basis of holomorphic one forms $\left\{w_{1}, \ldots, w_{g}\right\}$, that is, $\int_{\alpha_{i}} w_{j}=\delta_{i j}$.

The Siegel space $\mathcal{H}_{g}$ is the space consisting of the complex symmetric matrices of size $g \times g$ with positive imaginary part. The Riemann matrix $Z=\left(\int_{\beta_{j}} w_{i}\right)$ belongs to $\mathcal{H}_{g}$ as consequence of Riemann's bilinear relations.

Each matrix $Z=X+i Y \in \mathcal{H}_{g}$ produces a lattice $L_{Z}$ in $\mathbf{C}^{g}$ : the lattice generated by integer linear combinations of the columns of the period matrix $\left(\begin{array}{ll}I & Z\end{array}\right)$. Its Gram matrix is given by $H=P+i J$, where

$$
P=\left(\begin{array}{cc}
Y^{-1} & Y^{-1} X \\
X Y^{-1} & X Y^{-1} X+Y
\end{array}\right),
$$


defines a principal polarization on the complex torus $\mathbf{C}^{g} / L_{Z}$ and, in particular, we obtain a principally polarized Abelian variety.

The group $K$ induces a natural faithful representation $\theta: K \rightarrow$

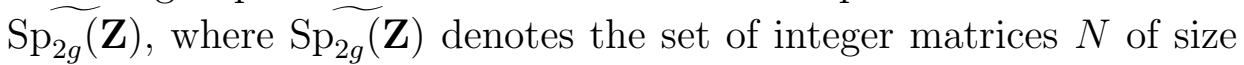
$2 g \times 2 g$ such that ${ }^{t} N J N= \pm J$ (the extended symplectic group). Its

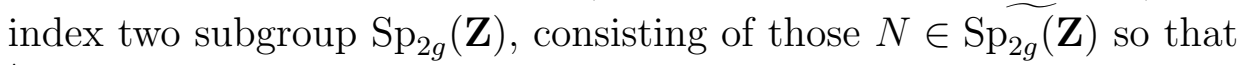
${ }^{t} N J N=J$, is called the symplectic group.

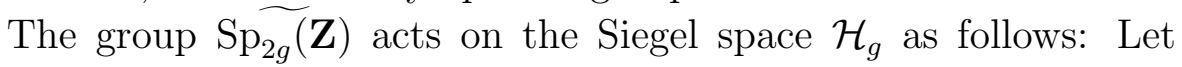
$Z \in \mathcal{H}_{g}$ and

$$
\begin{gathered}
N=\left(\begin{array}{cc}
A & B \\
C & D
\end{array}\right) \in \widetilde{\operatorname{Sp}_{2 g}(\mathbf{Z}),} \text { then } \\
N(Z)=\left\{\begin{array}{lll}
(A+Z C)^{-1}(B+Z D), & \text { if } & N \in \operatorname{Sp}_{2 g}(\mathbf{Z}) \\
(A+\bar{Z} C)^{-1}(B+\bar{Z} D), & \text { if } & N \notin \operatorname{Sp}_{2 g}(\mathbf{Z})
\end{array}\right.
\end{gathered}
$$

The matrix $Z=\left(\int_{\beta_{j}} w_{i}\right)$ is fixed by the symplectic transformation $\theta(k)$ for each $k \in K$.

For $Z \in \mathcal{H}_{g}$ fixed by the symplectic transformations $\theta(k)$, for all $k \in K$, the lattice $L_{Z}$ induced by $Z$ has $\theta(K)$ has group of isometries (for the positive Hermitian form $H$ ). In particular, they define principally polarized Abelian varieties with a group of automorphisms isomorphic to $K$.

Remark. If we have $Z=X+i Y \in \mathcal{H}_{g}$ and consider $Y^{-1}={ }^{t} B B$, then the lattice $L_{Z, B}=\left(\begin{array}{ll}B & B Z\end{array}\right)$ is isomorphic to $L_{Z}$. The Hermitian form in this new lattice is the standard one. A main problem is to determine those lattices for which the minimum non-zero norm is maximal [3] [22] [23]. One may try to use the explicit families in this work to consider the above extremal problem.

\section{Schottky Uniformizations and Riemann Matri- ces}

In this section we describe how Schottky uniformizations of Riemann automorphisms with a Schottky type automorphism group can be used to compute principally polarized Abelian varieties. 
Assume we have a Kleinian group $K$ (admitting anticonformal Moebius transformations) containing a Schottky group $J$ of genus $g \geq 2$, with region of discontinuity $\Omega$, as a normal subgroup of finite index. We have then that $K$ has also $\Omega$ as region of discontinuity [16].

Let us denote by $S$ the closed Riemann surface of genus $g$ uniformized by $G$, that is, $\Omega / G=S$, and denote by $P: \Omega \rightarrow S$ the natural holomorphic covering with $G$ as covering group.

Let us fix some fundamental set of loops for $J$, say $\alpha_{1}, \ldots, \alpha_{g}, \widetilde{\alpha}_{1}, \ldots$, $\widetilde{\alpha}_{g}$, and let $A_{1}, \ldots, A_{g}$ a set of free generators of $J$ respect to these loops.

We give orientation on the loops $\alpha_{1}, \ldots, \alpha_{g}$. Also we consider suitable oriented paths $\beta_{1}, \ldots, \beta_{g}$, pairwise disjoint, so that $\beta_{k}$ connects equivalent points in $\alpha_{k}$ and $\widetilde{\alpha}_{k}$, and $\beta_{k}$ is disjoint from $\alpha_{t}$ and $\widetilde{\alpha_{t}}$ for all $t \neq k$. Let us denote again by $\alpha_{k}$ and $\beta_{k}$ the projections on $S$ of the above respective loops. We have then that they define a symplectic base for $S$ and, in particular, a Riemann matrix $Z_{0}$ for $S$.

The group $K$ induces a finite group of conformal automorphisms on $S$, the group $K / G$. In particular, the choice of the symplectic

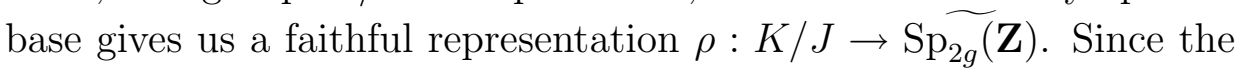
normalizer of the projected loops $\alpha_{1}, \ldots, \alpha_{g}$ is kept invariant under the action of $K / J$ (because of the lifting property of $K / J$ by $P: \Omega \rightarrow S$ ), we have that:

(1) if $\gamma \in K$ is conformal:

$$
\rho(\gamma)=\left(\begin{array}{cc}
\Theta(\gamma) & B_{\gamma} \\
0 & { }^{t} \Theta(\gamma)
\end{array}\right)
$$

(2) if $\gamma \in K$ is anti-conformal:

$$
\rho(\gamma)=\left(\begin{array}{cc}
\Theta(\gamma) & B_{\gamma} \\
0 & -{ }^{t} \Theta(\gamma)
\end{array}\right)
$$

where $\Theta: K / J \rightarrow G L(g, \mathbf{Z})$ is the isomorphism induced at the level of homology on the submodule generated by the loops $\alpha_{1}, \ldots, \alpha_{g}$. 
On the other hand, since $J$ is normal subgroup of $K$, we have that each $\gamma \in K$ induces an isomorphism $\eta(\gamma): J \rightarrow J$ and an isomorphism $\eta(\gamma)^{a b}: J /[J, J] \rightarrow J /[J, J]$. It is not hard to check that:

(1) if $\gamma \in K$ is conformal, then $\Theta(\gamma)={ }^{t} \eta(\gamma)^{a b}$; and

(2) if $\gamma \in K$ is ant-conformal, then $\Theta(\gamma)=-{ }^{t} \eta(\gamma)^{a b}$

\subsection{Schottky Uniformization With Invariant Circles}

In the particular case that $K$ keeps invariant an Euclidean circle $C$, the reflection $\sigma$ on $C$ will commute with every element of $K$. In particular, the surface $S$ uniformized by $J$ has an anticonformal involution with fixed points and commuting with each automorphism of $S$ induced by $K$. In this case, the above procedure asserts that $\eta(\sigma)^{a b}$ is the identity matrix, that is,

$$
\rho(\sigma)=\left(\begin{array}{cc}
-I & B_{\sigma} \\
0 & I
\end{array}\right)
$$

If $Z \in \mathcal{H}_{g}$ is fixed by the group $\rho(K / J)$, then in particular is fixed by $\rho(\sigma)$. This follows that $\operatorname{Re}(Z)=\frac{1}{2} B_{\sigma}$. From this also follows that:

(1) if $\gamma \in K$ is conformal, then $B(\gamma)=\frac{1}{2}\left(B(\sigma) \eta(\gamma)^{a b}-{ }^{t} \eta(\gamma)^{a b} B(\sigma)\right)$; and

(2) if $\gamma \in K$ is anti-conformal, then $B(\gamma)=\frac{1}{2}\left(B(\sigma) \eta(\gamma)^{a b}+{ }^{t} \eta(\gamma)^{a b} B(\sigma)\right)$

The computation of $B(\sigma)$ is simple in this case. In particular, we

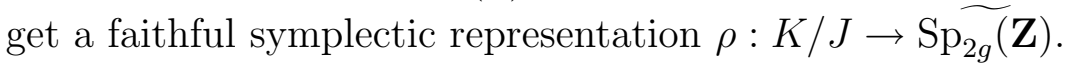

In sections 8 we use the constructed Schottky uniformizations to describe faithful symplectic representations of the groups $K(S, \tau)$ in the respective considered cases. We compute their fixed points in the respective Siegel space, that is, principally polarized Abelian varieties with group of automorphisms isomorphic to $K(S, \tau)$.

\subsection{Burnside's Remark [2]}

Let us assume that $G$ is a Schottky group keeping invariant some Euclidean circle on the Riemann sphere (a line is a circle through 
infinite) and for which $\infty \in \Omega(G)$. Denote by $G(\infty)$ the orbit of $\infty$. Burnside has remarked that the series

$$
\sum_{g \in G} g^{\prime}(z)
$$

converges uniformly on compact subsets of $\Omega(G)$ to a meromorphic map which is holomorphic on $\Omega(G)-G(\infty)$ and it has doble poles at each point in $G(\infty)$. The proof for the case that $G$ keeps invariant a line $L$ is rather simple. In fact, let $K \subset \Omega(G)$ be some compact set and $M=\left\{g_{1}, \ldots, g_{n}\right\} \subset G$ be so that $g_{j}^{-1}(\infty) \in K$. Then there is a positive constant $A>0$ so that

$$
\sum_{g \in G-M}\left|g^{\prime}(z)\right| \leq A \sum_{g \in G} \frac{1}{|c|^{2}}
$$

where $g(z)=\frac{a z+b}{c z+d}, a d-b c=1$.

Let us choose a point $q \in L$ and a positive number $R>1$ so that $B=\{z \in \mathcal{C}:|z-q|>R\} \cup\{\infty\}$ is precisely invariant under the identity in $G$. In this case, we have that the sets $g(B)$ are discs orthogonal to $L$ and, in particular,

$$
\sum_{g \in G, g \neq I} \operatorname{diam}(g(B)) \leq 2 R
$$

where diam denotes the Euclidean diameter. We may use the inequality given in C.7 of [15] which in our case reads

$$
\operatorname{diam}(g(B)) \geq \frac{1}{|c|^{2}} \frac{1}{\operatorname{dist}\left(g^{-1}(\infty), B\right)} \geq \frac{1}{|c|^{2}} \frac{1}{R},
$$

for $g \neq I$.

Since for $K \subset \Omega(G)-G(\infty)$ we have $M=\emptyset$, the above says that

$$
\sum_{g \in G} g^{\prime}(z)
$$

converges uniformly on $K$ to a holomorphic map. Now, around a point $g_{1}^{-1}(\infty)$ we have that the series

$$
\sum_{g \in G-\left\{g_{1}\right\}} g^{\prime}(z)
$$


converges locally to a holomorphic map and, since $g_{1}^{\prime}(z)$ has a doble pole in $g_{1}^{-1}(\infty)$, we are done in this case.

The case of circles can be carried to that situation in the following way. Assume that $C$ is the invariant circle. Choose some point $q \in$ $C \cap \Omega(G)$ (then $q \neq \infty)$. Let $T(z)=\frac{1}{z-q}$. Set $H=T G T^{-1}$ and $L=T(S)$. Then $H$ is a Schottky group keeping invariant the line $L$ and for which $\infty \in \Omega(H)$. In this case we have the convergence property for $\sum_{h \in H} h^{\prime}(w)$ on compact sets of $\Omega(H)$. Let $K$ be a compact subset of $\Omega(G)$ so that $q \notin K$. Then for $z \in K$ we have

$$
\sum_{h \in H, h \neq I}\left|h^{\prime}(T(z))\right|=\sum_{g \in G, g \neq I}\left|g^{\prime}(z)\right| \frac{1}{|g(z)-q|^{2}|T(z)|^{2}} .
$$

The fact that $q \notin K$ asserts that

$$
\operatorname{Inf}\left\{\frac{1}{|T(z)|^{2}} ; z \in K\right\}=M>0 .
$$

Also, except for a finite number of elements of $G$, say $g_{1}, \ldots, g_{n}$, we have that

$$
\operatorname{Inf}\left\{\frac{1}{|g(z)-q|^{2}} ; z \in K, g \in G-\left\{g_{1}, \ldots, g_{n}\right\}\right\}=N>0 .
$$

Now it follows that

$$
\sum_{g \in G, g \neq I}\left|g^{\prime}(z)\right| \frac{1}{|g(z)-q|^{2}|T(z)|^{2}} \geq N M \sum_{g \in G, g \neq I}\left|g^{\prime}(z)\right|,
$$

obtaining the desired convergence.

As a consequence of the above, we have the following. Let us consider a set of free generators $A_{1}, \ldots, A_{g}$ for the Schottky group $G$. Let $\alpha_{1}, \ldots, \alpha_{g}, \widetilde{\alpha_{1}}, \ldots, \widetilde{\alpha_{g}}$ be a set of simple loops defining a standard fundamental domain for $G$, respect to the above generators. If we give the counterclockwise orientation to the loops $\alpha_{j}$, then

$$
w_{j}(z)=\frac{1}{2 \pi i} \sum_{g \in G} \frac{g^{\prime}(z)}{g(z)-A_{j}^{-1}(\infty)} d z
$$

for $j=1, \ldots, g$, are the lifting to $\Omega(G)$ of the holomorphic one-forms dual to the homological half-symplectic basis on $S=\Omega(G) / G$ determined by the projections of the oriented loops $\alpha_{1}, \ldots, \alpha_{g}$. 


\section{Maximal Symmetric Riemann Surfaces of genus 5}

Let us consider a maximal symmetric Riemann surface $S$ of genus five together a maximal reflection $\tau: S \rightarrow S$ so that $S / \tau$ is orientable. We already noted that $S / K(S, \tau)$ is the closed disc with exactly four branch values of orders $2,2,2$ and 3 in its border.

Theorem 5.1. In genus 5 there are exactly two different topological maximal reflections $\tau$ with the condition that $S / \tau$ is orientable. They give rise to topologically rigid and non-isomorphic groups $K(S, \tau)$. In particular, we have exactly two algebraic real sets in the moduli space of genus five, each one of real dimension one, determining the surfaces above in consideration. In each case, there is a normal subgroup $L$ of $K(S, \tau)$, isomorphic to $\mathcal{Z}_{2}+\mathcal{Z}_{2}$, of conformal automorphisms acting freely and $S / L$ is a closed Riemann surface of genus two.

Proof. Since $S / \tau$ is orientable, we have exactly three possibilities:

(1) $S / \tau$ is a genus one Riemann surface with four boundary components;

(2) $S / \tau$ is a genus zero Riemann surface with six boundary components; and

(3) $S / \tau$ is a genus two Riemann surface with two boundary components.

The fact that $\tau$ belong to the centralizer of $K(S, \tau)$, we have that $K(S, \tau)$ descends to a group of automorphisms $H_{48}$, of order 48, on the quotient $S / \tau$.

(1) $S / \tau$ has genus one and four boundary components. Denote by $H_{48}$ the group of order 48 of automorphisms of $S / \tau$ induced by $H_{96}$. If $H_{48}$ only has conformal automorphisms, then this will imply the existence of a group of conformal automorphisms of order 48 acting on a genus one Riemann surface keeping transitively invariant a set of 4 points. This implies that the stabilizer in such group of any of these points is a 
cyclic group of order 12, which is impossible. It follows that $H_{48}$ must have orientation reversing automorphisms. Let $H_{24}$ be the index two subgroup of orientation preserving automorphisms of $H_{48}$. The action of such a group corresponds (topologically) to the action of a conformal group of order 24 on a genus one Riemann surface with four punctures. This action is the unique action of the group generated by an alternating group $\mathcal{A}_{4}=\langle W, T\rangle$ and an involution $J$. Each puncture is fixed by $J$. In this case, the genus one surface corresponds to the curve $y^{2}=x^{3}-1$ and the group $H_{48}$ is generated by the transformations $J:(x, y) \rightarrow(x,-y), S:(x, y) \rightarrow(\bar{x}, \bar{y}), T:(x, y) \rightarrow$ $\left(\frac{x+2}{x-1}, \frac{3 y}{(x-1)^{2}}\right)$ and $W:(x, y) \rightarrow(\rho x, y)$, where $\rho=e^{\frac{2 \pi i}{3}}$, where the group $H_{24}$ is the alternating group generated by $W$ and $T$. In particular, the topological action of $K(S, \tau)$ is unique in this situation and has presentation: $K(S, \tau)=\langle T, W, J, \tau$ : $T^{2}=W^{3}=J^{2}=\tau^{2}=(T W)^{2}=(T J)^{2}=(T \tau)^{2}=\tau W \tau W^{-1}=$ $\left.(\tau J)^{2}=\left(J W J W^{-1}\right)^{2}=(\tau W)^{6}=1\right\rangle$.

Let us observe at this point that $K(S, \tau)$ has to involutions (the corresponding to $J$ and $\tau S T$ ) generate the Klein group $L=\mathcal{Z}_{2}+\mathcal{Z}_{2}$ acting free fixed points, which is normal subgroup. The surface $S / L$ is a genus two Riemann surface with group of automorphisms $\mathcal{Z}_{2}+\mathcal{Z}_{2}+D_{3}$, where $D_{3}$ denotes a dihedral group of order 6 . One of the $\mathcal{Z}$ is a reflection (induced by $\tau$ ) with exactly one component of fixed points, a dividing closed simple geodesic $\gamma$. Such a closed geodesic divides $S / L$ into two one holed tori, say $T_{1}$ and $T_{2}$. The other $\mathcal{Z}_{2}$ is the hyperelliptic involution and $D_{3}$ is the dihedral group generated by an automorphisms of order 3 keeping invariant each $T_{i}$ and a conformal involution permuting $T_{1}$ and $T_{2}$. In section 4 we produce the Schottky uniformizations of these surfaces of genus two.

(2) $S / \tau$ is the Riemann sphere with six boundary compo-

nents. Let $H_{48}$ be the induced group of automorphisms by $H_{96}$ on this surface. If $H_{48}$ only has conformal automorphisms, then it implies the existence of a group of Möbius transformations of order 48 keeping transitively invariant a set of 6 points, which is impossible. It follows that $H_{48}$ has anticonformal automor- 
phisms. Let $H_{24}$ be the index two subgroup of $H_{48}$ of conformal automorphisms. Topologically, this corresponds to the action of the symmetric group $\mathcal{S}_{4}=\left\langle a, b: a^{4}=b^{2}=(a b)^{3}=1\right\rangle$, where the six special points are exactly the six fixed points of the elements of order 4. The extra anticonformal automorphism is in fact a reflection which fixes four of these points and interchange the other two. In particular, the topological action of $K(S, \tau)$ is unique and has presentation: $K(S, \tau)=\left\langle a, b, \mu, \tau: a^{4}=b^{2}=\mu^{2}=\tau^{2}=\right.$ $\left.(a b)^{3}=\tau a \tau a^{-1}=(\tau b)^{2}=(\mu \tau)^{2}=\mu a \mu a^{-1}=\mu b \mu a^{2} b a^{2}=1\right\rangle$.

The subgroup $L=\left\langle a^{2}, b a^{2} b\right\rangle \cong \mathcal{Z}_{2}+\mathcal{Z}_{2}$ is a normal subgroup of $K(S, \tau)$ acting freely. The quotient surface $S / L$ is a genus two Riemann surface admitting the group $\mathcal{Z}_{2}+\mathcal{Z}_{2}+D_{3}$. In this case, one of the $\mathcal{Z}_{2}$ is generated by a reflection (the one induced by $\tau$ ) with exactly three components of fixed points (an M-symmetry). These three components divide $S / L$ into two three holed spheres, say $S_{1}$ and $S_{2}$. The other $\mathcal{Z}_{2}$ is generated by the hyperelliptic involution and $D_{3}$ is generated by an automorphism of order three keeping invariant each $S_{i}$ and a conformal involution also keeping invariant each $S_{i}$. In section 4 we produce the Schottky uniformizations of these surfaces of genus two.

(3) $S / \tau$ is a genus two Riemann surface with two boundary components. Again, we denote by $H_{48}$ the induced automorphism group. Either this has only conformal automorphisms or there is an index two subgroup of conformal automorphisms. In any of this cases, it will imply the existence of a group of conformal automorphisms of either order 48 or 24 on a closed Riemann surface of genus two keeping invariant two points. The stabilizer of any of this points will be either a cyclic group of order 24 or 12 , which is impossible.

In any of the two possible cases (1) and (2), the family depends on one real dimensional parameter. This because the Teichmüller space of the closed unit disc with four marked points on the boundary has real dimension one.

We must remark at this point that in genus two, there are exactly two different topological actions of a group $\mathcal{Z}_{2}+\mathcal{Z}_{2}+D_{3}$ as group 
of automorphisms where one of the $\mathcal{Z}_{2}$ is generated by a reflection. These two are the ones described in (1) and (2), respectively.

\section{Uniformization of the closed disc with branch values of orders 2, 2, 2 and 3 on its border}

For each $p \in(2-\sqrt{3}, 1)$, let us consider the group $\widehat{K}$ generated by the following reflections: $\sigma_{1}(z)=\bar{z}, \sigma_{2}(z)=e^{\frac{2 \pi i}{3}} \bar{z}, \sigma_{p}(z)=\frac{\left(1+p^{2}\right) \bar{z}-2 p}{2 p \bar{z}-\left(1+p^{2}\right)}$ and $\sigma(z)=\frac{1}{\bar{z}}$ (see figure 1 ).

If we set $W(z)=\sigma_{2} \sigma_{1}(z)=e^{\frac{2 \pi i}{3}} z, T(z)=\sigma \sigma_{1}(z)=\frac{1}{z}$ and

$$
J(z)=\sigma_{p} \sigma_{1}(z)=\frac{\left(p+\frac{1}{p}\right) z-2}{2 z-\left(p+\frac{1}{p}\right)},
$$

then the group $\widehat{K}$ is also generated by the transformations $T, W, J$ and $\sigma$. As consequence of Klein-Maskit's combination theorems [15], we have

$$
\begin{gathered}
\widehat{K}=\left\langle T, W, J, \sigma: T^{2}=W^{3}=(W T)^{2}=J^{2}=(T J)^{2}=\sigma^{2}=(\sigma T)^{2}=\right. \\
\left.\sigma W \sigma W^{-1}=(\sigma J)^{2}=1\right\rangle .
\end{gathered}
$$

The Kleinian group $\widehat{K}$ has a connected region of discontinuity $\Omega$ and a fundamental domain for it is determined the the bounded region determined by the unit circle, the two rays of arguments $\frac{\pi i}{3}$ and $\frac{-\pi i}{3}$, respectively, and the circle $C$ orthogonal to the unit circle and containing the points $p$ and $\frac{1}{p}$ (see figure 1 ). The Klein surface uniformized by $\widehat{K}$ is the closed unit disc with exactly four branch values of orders 2, 2, 2 and 3 on its border. 


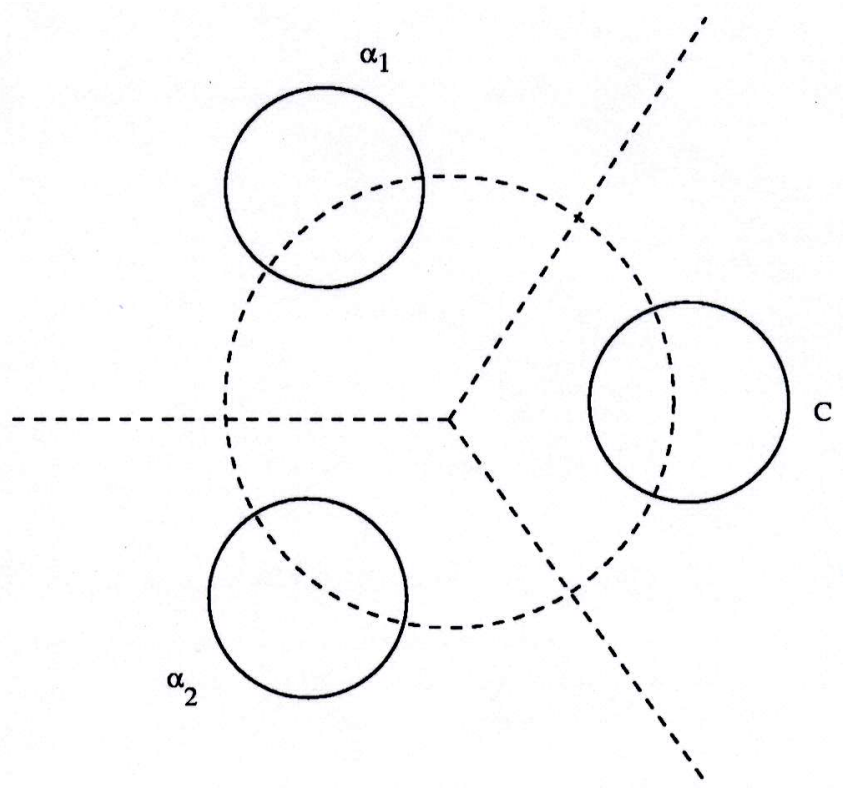

Figure 1

Theorem 6.1. Every Klein surface which is the unit closed disc with exactly four branch values of orders 2, 2, 2 and 3 on the border can be uniformized by a group $\widehat{K}$ for some $p \in(2-\sqrt{3}, 1)$.

Proof. This is just consequence of quasiconformal deformation and the fact that every reflection on the Riemann sphere has an Euclidean circle as fixed points.

The index two subgroup $K$ of $\widehat{K}$ of the orientation preserving transformations is generated by the transformations $T, W$ and $J$ and the presentation of $K$ is

$$
K=\left\langle T, W, J: T^{2}=W^{3}=(W T)^{2}=J^{2}=(T J)^{2}=1\right\rangle,
$$


that is, isomorphic to the free amalgamated product of the dihedral group $D_{3}=\langle T, W\rangle$ with the Klein group $\mathcal{Z}_{2}+\mathcal{Z}_{2}=\langle T, J\rangle$ over the cyclic group of order two generated by $T$. The Riemann surface $\Omega / K$ is the Riemann sphere with four branched values of order 2, 2, 2 and 3, all of them fixed by the reflection induced by $\sigma$ on it. A fundamental domain of $K$ can be considered as the union of the above fundamental domain of $\widehat{K}$ with its image under $\sigma$ together the points of the unit circle located between $e^{\frac{\pi i}{3}}$ and $e^{\frac{-\pi i}{3}}$, respectively.

Remark. (1) The boundary case $p=2-\sqrt{3}$ corresponds to the case that $\widehat{K}$ uniformizes a hyperbolic closed triangle with one vertex at infinity, one vertex with angle $\frac{\pi}{3}$ and the other with angle $\frac{\pi}{2}$. The corresponding group $K$ uniformizes an sphere with a puncture and two branched values of orders 2 and 3, respectively. (2) The boundary case $p=1$ corresponds to the case that $\widehat{K}$ is only generated by the reflections $\sigma_{1}, \sigma_{2}$ and $\sigma$, but acting on the three-punctured Riemann sphere. In this case, the group $\widehat{K}$ uniformizes the closed unit disc with three branched values at the border with orders 2,3 and $\infty$, respectively.

\section{Schottky uniformizations of the genus two max- imal symmetric Riemann surfaces}

Let us consider the Möbius transformations $A_{1}=J W J W^{-1}, A_{2}=$ $J W^{-1} J W$,

$B_{1}=\left(\sigma_{3} \sigma_{2}\right)^{2}$ and $B_{2}=\sigma_{1} B_{1} \sigma_{1}$. The groups $G_{2}=\left\langle A_{1}, A_{2}\right\rangle$ and $F_{2}=$ $\left\langle B_{1}, B_{2}\right\rangle$ are classical Schottky groups of genus two and a standard fundamental domain for both $G_{2}$ and $F_{2}$ is given by the circles $W(C)$, $J(W(C)), W^{-1}(C)$ and $J\left(W^{-1}(C)\right)$.

Since $W A_{1} W^{-1}=A_{1}^{-1} A_{2}, W A_{2} W^{-1}=A_{1}^{-1}, J A_{1} J=A_{1}^{-1}, J A_{2} J=$ $A_{2}^{-1}, T A_{1} T^{-1}=A_{2}, T A_{2} T=A_{1}, \sigma A_{1} \sigma=A_{1}, \sigma A_{2} \sigma=A_{2}, W B_{1} W^{-1}=$ $B_{1}^{-1} B_{2}, W B_{2} W^{-1}=B_{1}^{-1}, J B_{1} J=B_{2}^{-1}, J B_{2} J=B_{1}^{-1}, T B_{1} T=B_{2}$, $T B_{2} T=B_{1}, \sigma B_{1} \sigma=B_{1}$ and $\sigma B_{2} \sigma=B_{2}$, we have that both $G_{2}$ and $F_{2}$ are normal subgroups of $\widehat{K}$. Moreover, for $Q \in\left\{G_{2}, F_{2}\right\}$, $\widehat{K} / Q \cong D_{3}+\mathcal{Z}_{2}+\mathcal{Z}_{2}$ and $K / Q \cong D_{3}+\mathcal{Z}_{2}$. In particular, the Riemann surfaces of genus two $S_{2}=\Omega / G_{2}$ (respectively, $R_{2}=\Omega / F_{2}$ ) admit the group $\widehat{K} / G_{2}$ (respectively, $\widehat{K} / F_{2}$ ) as group of symmetries, with $K / G_{2}$ 
(respectively, $K / F_{2}$ ) as its index two subgroup of orientation preserving symmetries. The quotient $S_{2} /\left(\widehat{K} / G_{2}\right)$ (respectively, $R_{2} /\left(\widehat{K} / F_{2}\right)$ ) is the unit closed disc with four branch values in its boundary of orders 2, 2,2 and 3 .

Theorem 7.1. If $X$ is a maximal symmetric Riemann surface of genus two with a maximal reflection $\rho: X \rightarrow X$ so that $X / \rho$ is orientable, then it can be uniformized by either $G_{2}$ or $F_{2}$ for a suitable $p \in(2-\sqrt{3}, 1)$.

Proof. Fix a value of $p \in(2-\sqrt{3}, 1)$ and let $K$ be the corresponding group above constructed. Let $X$ be a maximal symmetric Riemann surface of genus two together a maximal reflection $\tau: X \rightarrow X$ as in the hypothesis. The topological action of $K(X, \tau)$ is either reflected by $G_{2}$ or $F_{2}$, since there are exactly two possible actions. Without lost of generality, we may assume that this is reflected by $G_{2}$. Set $S_{2}=\Omega / G_{2}$ and let $f: S_{2} \rightarrow X$ be an orientation preserving homeomorphisms so that $f \widehat{K} / G_{2} f^{-1}=K(X, \tau)$. We may assume that $f$ is quasiconformal homeomorphisms with complex Beltrami differential $\mu$. We lift $\mu$ to $\Omega$ and extend it as zero to the limit set of $\widehat{K}$. Let $W: \widehat{\mathcal{C}} \rightarrow \widehat{\mathcal{C}}$ be a $\mu$-quasiconformal homeomorphisms. Then $W \widehat{K} W^{-1}$ is again a group generated by reflections. We may assume that $W$ fixes 0,1 and $\infty$. Since a reflection has as fixed points only Euclidean circles on the Riemann sphere, we have that the group $W \widehat{K} W^{-1}$ is again one of our groups $\widehat{K}$ for a suitable value of $p$. If we denote by $\pi: \Omega \rightarrow \Omega / G_{2}$ the holomorphic covering induced by $G_{2}$, then we have that $f \pi W^{-1}: \Omega\left(W \widehat{K} W^{-1}\right) \rightarrow X$ an uniformization of $X$ by the corresponding Schottky group $G_{2}$, so that the lifting of $K(X, \tau)$ is exactly $W \widehat{K} W^{-1}$.

\subsection{The Schottky Group $G_{2}$}

In this subsection we proceed to see the following:

Theorem 7.2. Let $X$ be a maximal symmetric Riemann surface of genus 2 with a maximal reflection $\rho: X \rightarrow X$ so that $X / \rho$ is a genus one surface with one hole. Let $p \in(2-\sqrt{3}, 1)$ be so that $X=\Omega / G_{2}$. 
Then a Riemann matrix of $X$ is given by

$$
Z=\frac{1}{2}\left[\begin{array}{ll}
0 & 1 \\
1 & 0
\end{array}\right]+i\left[\begin{array}{cc}
w & \frac{w}{2} \\
\frac{w}{2} & w
\end{array}\right],
$$

where

$$
\begin{gathered}
w=\frac{1}{2 \pi} \log \left(\prod_{\gamma \in G_{2}} \frac{\gamma\left(q_{2}\right)-A_{1}^{-1}(\infty)}{\gamma\left(q_{1}\right)-A_{1}^{-1}(\infty)}\right), \\
q_{1}=e^{\left(\frac{2 \pi}{3}-\theta\right) i}, \quad q_{2}=J\left(q_{1}\right)
\end{gathered}
$$

and $\theta \in\left(0, \frac{\pi}{2}\right)$ is such that $\cos \theta=\frac{2 p}{1+p^{2}}$.

Proof. Set $\alpha_{1}=W(C), \alpha_{1}^{\prime}=J\left(\alpha_{1}\right), \alpha_{2}=W^{-1}(C)$ and $\alpha_{2}^{\prime}=$ $J\left(\alpha_{2}\right)$. If we orient the circles $\alpha_{1}, \alpha_{2}, \alpha_{1}^{\prime}$ and $\alpha_{2}^{\prime}$ and consider the oriented paths $\beta_{1}$ and $\beta_{2}$ as shown in figure 2, then the projection of these loops and paths determine a symplectic basis on the closed Riemann surface $S_{2}$. 

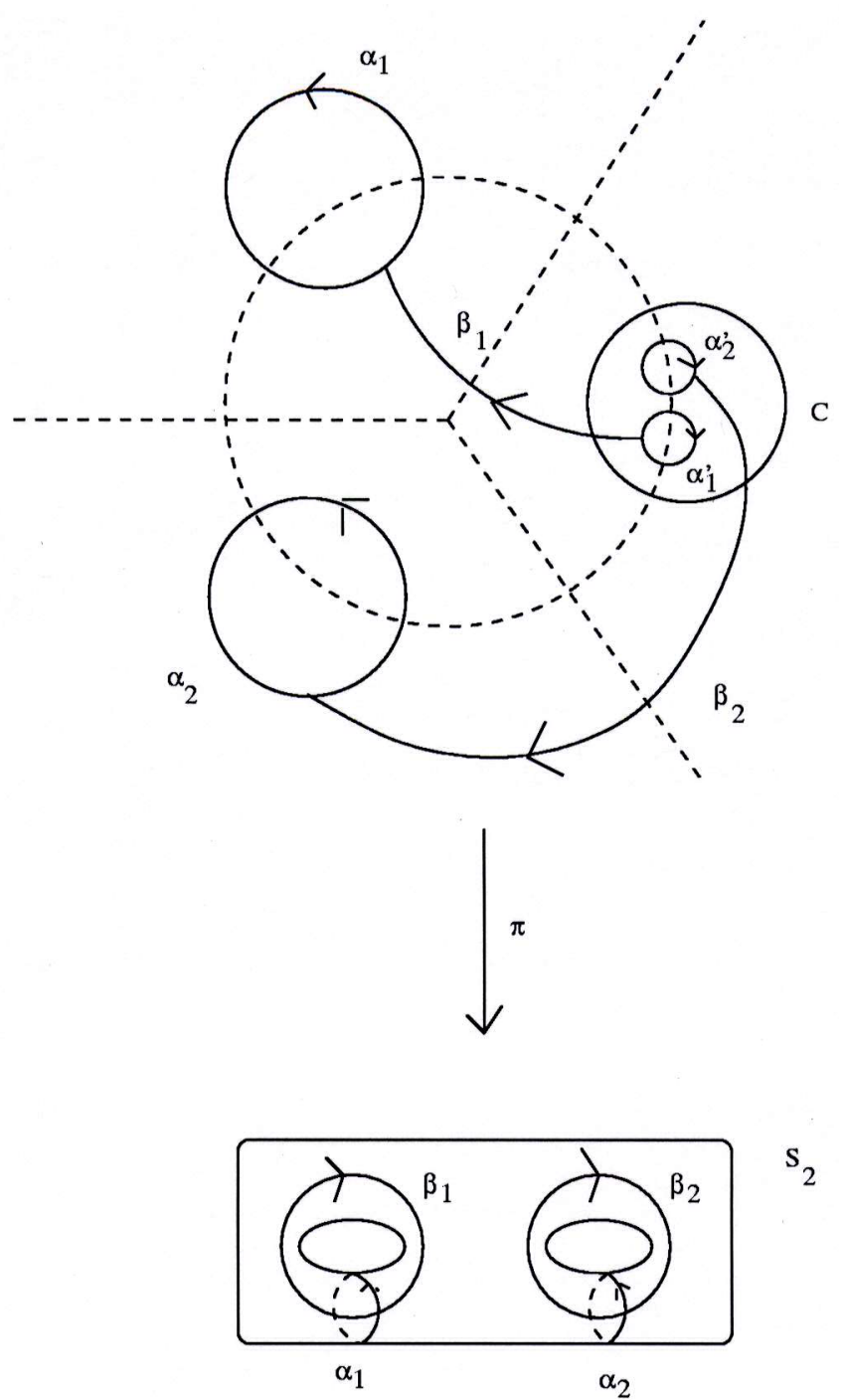

Figure 2

In this way, we obtain a faithful symplectic representation

$$
\rho: \widehat{K} / G_{2} \rightarrow \operatorname{Sp} \widetilde{(4 ; \mathcal{Z})}
$$


defined by

$$
\begin{gathered}
\rho(\sigma)=\left[\begin{array}{rrrr}
-1 & 0 & 0 & -1 \\
0 & -1 & -1 & 0 \\
0 & 0 & 1 & 0 \\
0 & 0 & 0 & 1
\end{array}\right], \quad \rho(T)=\left[\begin{array}{llll}
0 & 1 & 0 & 0 \\
1 & 0 & 0 & 0 \\
0 & 0 & 0 & 1 \\
0 & 0 & 1 & 0
\end{array}\right] \\
\rho(J)=\left[\begin{array}{rrrr}
-1 & 0 & 0 & 0 \\
0 & -1 & 0 & 0 \\
0 & 0 & -1 & 0 \\
0 & 0 & 0 & -1
\end{array}\right], \quad \rho(W)=\left[\begin{array}{rrrr}
0 & -1 & a & b \\
1 & -1 & c & d \\
0 & 0 & -1 & -1 \\
0 & 0 & 1 & 0
\end{array}\right]
\end{gathered}
$$

The Riemann matrix $Z=X+i Y \in \mathcal{H}_{2}$ of $S_{2}$ defined by the above symplectic basis is a fixed point of the symplectic group $\rho\left(\widehat{K} / G_{2}\right)$. It follows that: $a=-1, b=0, c=0, d=1$, and

$$
Z=\frac{1}{2}\left[\begin{array}{ll}
0 & 1 \\
1 & 0
\end{array}\right]+i\left[\begin{array}{cc}
w & \frac{w}{2} \\
\frac{w}{2} & w
\end{array}\right],
$$

where $w>0$.

The results due to Burnside [2] described at the end of section 4 can be used in this case to the group $G_{2}$ to compute the value of $w$ in function of $p$ as described in the theorem.

Remark. In the case $p=2-\sqrt{3}$, the group $G_{2}$ is a noded Schottky group of genus two uniformizing an stable Riemann surface of genus two with exactly one dividing node. In the case $p=1$, we can think of the group $G_{2}$ as the trivial group acting on the three-punctured sphere, that is, uniformizing the three-punctured sphere.

\subsection{The Schottky Group $F_{2}$}

In this subsection we proceed to see the following:

Theorem 7.3. Let $X$ be a maximal symmetric Riemann surface of genus 2 with a maximal reflection $\rho: X \rightarrow X$ so that $X / \rho$ is a genus zero surface with three holes. Let $p \in(2-\sqrt{3}, 1)$ be so that $X=\Omega / F_{2}$. Then a Riemann matrix of $X$ is given by

$$
Z=i\left[\begin{array}{cc}
w & \frac{w}{2} \\
\frac{w}{2} & w
\end{array}\right]
$$


where

$$
\begin{gathered}
w=\frac{1}{2 \pi} \log \left(\prod_{\gamma \in F_{2}} \frac{\gamma\left(p_{2}\right)-B_{1}^{-1}(\infty)}{\gamma\left(q_{1}\right)-B_{1}^{-1}(\infty)}\right), \\
q_{1}=e^{\left(\frac{2 \pi}{3}-\theta\right) i}, \quad p_{2}=\sigma_{3}\left(q_{1}\right)
\end{gathered}
$$

and $\theta \in\left(0, \frac{\pi}{2}\right)$ is such that $\cos \theta=\frac{2 p}{1+p^{2}}$.

Proof. Set $\alpha_{1}=W(C), \alpha_{1}^{\prime}=\sigma_{3}\left(\alpha_{1}\right), \alpha_{2}=W^{-1}(C)$ and $\alpha_{2}^{\prime}=$ $\sigma_{3}\left(\alpha_{2}\right)$. If we orient the circles $\alpha_{1}, \alpha_{2}, \alpha_{1}^{\prime}$ and $\alpha_{2}^{\prime}$ and consider the oriented paths $\beta_{1}$ and $\beta_{2}$ as shown in figure 3 , then the projection of these loops and paths determine a symplectic basis on the closed Riemann surface $R_{2}$.

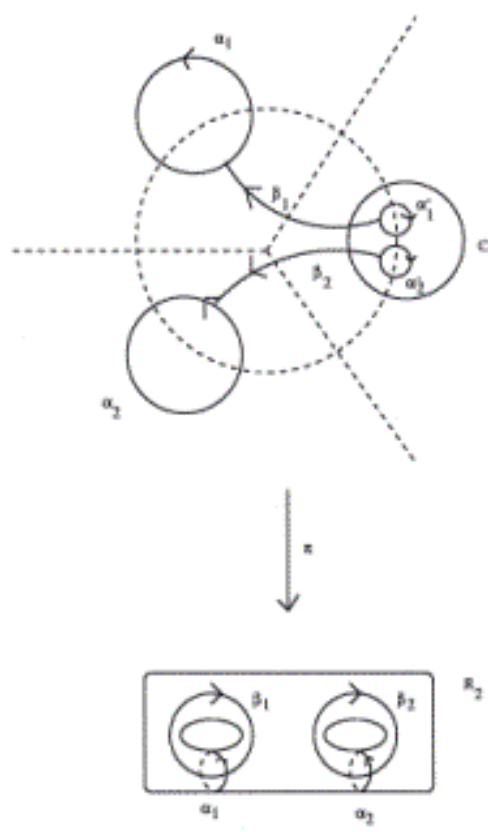

Figure 3 
In this way, we obtain a faithful symplectic representation

$$
\left.\rho: \widehat{K} / F_{2} \rightarrow \operatorname{Sp} \widetilde{(4 ;} \mathcal{Z}\right)
$$

defined by

$$
\begin{gathered}
\rho(\sigma)=\left[\begin{array}{rrrr}
-1 & 0 & 0 & 0 \\
0 & -1 & 0 & 0 \\
0 & 0 & 1 & 0 \\
0 & 0 & 0 & 1
\end{array}\right], \quad \rho(T)=\left[\begin{array}{llll}
0 & 1 & 0 & 0 \\
1 & 0 & 0 & 0 \\
0 & 0 & 0 & 1 \\
0 & 0 & 1 & 0
\end{array}\right] \\
\rho(J)=\left[\begin{array}{rrrr}
0 & -1 & 0 & 0 \\
-1 & 0 & 0 & 0 \\
0 & 0 & 0 & -1 \\
0 & 0 & -1 & 0
\end{array}\right], \quad \rho(W)=\left[\begin{array}{rrrr}
0 & -1 & a & b \\
1 & -1 & c & d \\
0 & 0 & -1 & -1 \\
0 & 0 & 1 & 0
\end{array}\right]
\end{gathered}
$$

The Riemann matrix $Z=X+i Y \in \mathcal{H}_{2}$ of $R_{2}$ defined by the above symplectic basis is a fixed point of the symplectic group $\rho\left(\widehat{K} / F_{2}\right)$. It follows that: $a=b=c=d=0$, and

$$
Z=i\left[\begin{array}{cc}
w & \frac{w}{2} \\
\frac{w}{2} & w
\end{array}\right]
$$

where $w>0$.

Again the results due to Burnside [2] described at the end of section 4 can be used in this case to the group $F_{2}$ to compute the value of $w$ in function of $p$, as desired.

\section{Remarks.}

(1) In the case $p=2-\sqrt{3}$, the group $F_{2}$ is a noded Schottky group of genus two uniformizing an stable Riemann surface of genus two with exactly three non-dividing nodes. In the case $p=1$, we can think of the group $F_{2}$ as the trivial group acting on the three-punctured sphere, that is, uniformizing the threepunctured sphere.

(2) Let us note at this point that the results in [20] (see also the work of González-Díez [6]) permit us to describe the algebraic curve 
(using theta functions) of the surfaces $S_{2}$ and $R_{2}$ in function of $w$ and, in particular, of $p$. In this way, we can obtain an explicit relation between the Schottky groups $G_{2}, F_{2}$ and the respective algebraic curves.

\section{Schottky uniformizations of the genus five max- imal symmetric Riemann surfaces}

Let $G_{5}$ (respectively, $F_{5}$ ) be the subgroup of $G_{2}$ (respectively, $F_{2}$ ) generated by the square of all the elements in $G_{2}$ (respectively, $F_{2}$ ), that is:

$$
\begin{aligned}
& G_{5}=\left\langle x^{2}: x \in G_{2}\right\rangle \\
& F_{5}=\left\langle x^{2}: x \in F_{2}\right\rangle .
\end{aligned}
$$

Since $G_{2}$ (respectively, $F_{2}$ ) is normal subgroup of index 24 in $\widehat{K}$ and $G_{5}$ (respectively, $F_{5}$ ) is normal of index 4 in $G_{2}$ (respectively, $F_{2}$ ), we have that $G_{5}$ (respectively, $F_{5}$ ) is a normal subgroup of $\widehat{K}$ of index 96. The group $G_{5}$ (respectively, $F_{5}$ ) is a free group of index four in the Schottky group $G_{2}$ (respectively, $F_{2}$ ) and then it is a Schottky group of genus 5 . Free generators for $G_{5}$ (respectively, $F_{5}$ ) are given by $D_{1}=A_{1}^{2}, D_{2}=A_{2}^{2}, D_{3}=A_{1}^{-1} A_{2}^{2} A_{1}, D_{4}=A_{1}^{-1} A_{2}^{-1} A_{1} A_{2}$ and $D_{5}=A_{2}^{-1} A_{1} A_{2} A_{1}$ (respectively, $E_{1}=B_{1}^{2}, E_{2}=B_{2}^{2}, E_{3}=B_{1}^{-1} B_{2}^{2} B_{1}$, $E_{4}=B_{1}^{-1} B_{2}^{-1} B_{1} B_{2}$ and $\left.E_{5}=B_{2}^{-1} B_{1} B_{2} B_{1}\right)$.

Theorem 8.1. If $S$ is a maximal symmetric Riemann surface of genus five admitting a maximal reflection $\tau: S \rightarrow S$ so that $S / \tau$ is orientable, then it can be uniformized by either $G_{5}$ or $F_{5}$ for a suitable $p \in(2-\sqrt{3}, 1)$.

Proof. The proof is exactly the same as done for theorem 7.1.

Remark. In the case $p=2-\sqrt{3}$, the group $G_{5}$ is a noded Schottky group uniformizing a stable Riemann surface of genus five with exactly four non-dividing nodes and two components, each one of genus one. Similarly, the group $F_{5}$ is a noded Schottky group uniformizing a stable Riemann surface of genus five with exactly six non-dividing nodes. 
A standard fundamental domain for $G_{5}$ (respectively, $F_{5}$ ) respect to the above free generators is determined by the circles $\theta_{1}=A_{1}^{-1}\left(\alpha_{1}\right)$, $\theta_{1}^{\prime}=\alpha_{1}^{\prime}, \theta_{2}=A_{2}^{-1}\left(\alpha_{2}\right), \theta_{2}^{\prime}=\alpha_{2}^{\prime}, \theta_{3}=A_{1}^{-1} A_{2}^{-1}\left(\alpha_{2}\right), \theta_{3}^{\prime}=A_{1}^{-1}\left(\alpha_{2}^{\prime}\right)$, $\theta_{4}=A_{2}^{-1}\left(\alpha_{1}\right), \theta_{4}^{\prime}=A_{1}^{-1} A_{2}^{-1}\left(\alpha_{1}^{\prime}\right), \theta_{5}=A_{1}^{-1} A_{2}^{-1}\left(\alpha_{1}\right)$ and $\theta_{5}^{\prime}=A_{2}^{-1}\left(\alpha_{1}^{\prime}\right)$ (respectively, $\theta_{1}=B_{1}^{-1}\left(\alpha_{1}\right), \theta_{1}^{\prime}=\alpha_{1}^{\prime}, \theta_{2}=B_{2}^{-1}\left(\alpha_{2}\right), \theta_{2}^{\prime}=\alpha_{2}^{\prime}, \theta_{3}=$ $B_{1}^{-1} B_{2}^{-1}\left(\alpha_{2}\right), \theta_{3}^{\prime}=B_{1}^{-1}\left(\alpha_{2}^{\prime}\right), \theta_{4}=B_{2}^{-1}\left(\alpha_{1}\right), \theta_{4}^{\prime}=B_{1}^{-1} B_{2}^{-1}\left(\alpha_{1}^{\prime}\right), \theta_{5}=$ $B_{1}^{-1} B_{2}^{-1}\left(\alpha_{1}\right)$ and $\left.\theta_{5}^{\prime}=B_{2}^{-1}\left(\alpha_{1}^{\prime}\right)\right)$.

We can give to these $\theta$-loops the orientations determined by the orientations we have given to $\alpha_{1}, \alpha_{2}, \alpha_{1}^{\prime}$ and $\alpha_{2}^{\prime}$. Let us choose the following paths: $\delta_{1}=\beta_{1} \cup A_{1}^{-1}\left(\beta_{1}\right), \delta_{2}=\beta_{2} \cup A_{2}^{-1}\left(\beta_{2}\right), \delta_{3}=A_{1}^{-1}\left(\delta_{2}\right)$ (respectively, $\delta_{1}=\beta_{1} \cup B_{1}^{-1}\left(\beta_{1}\right), \delta_{2}=\beta_{2} \cup B_{2}^{-1}\left(\beta_{2}\right), \delta_{3}=B_{1}^{-1}\left(\delta_{2}\right)$ ), with the orientations determined by the orientations given to $\beta_{1}$ and $\beta_{2}$. For $j=4,5$, we choose oriented paths $\delta_{j}$ contained inside the unit disc starting at $\theta_{j}$ and ending at $\theta_{j}^{\prime}$ and disjoint from $\delta_{1}$ and $\delta_{9-j}$. The projections of the above loops and paths determine on the Riemann surface $S_{5}$ (respectively, $R_{5}$ ) a symplectic basis. Since we have the following relations:

(a) $J D_{1} J=D_{1}^{-1}, J D_{2} J=D_{2}^{-1}, J D_{3} J=D_{1} D_{3}^{-1} D_{1}^{-1}, J D_{4} J=$ $D_{1} D_{3} D_{5}^{-1} D_{2}^{-1}, J D_{5} J=D_{2} D_{4}^{-1} D_{3}^{-1} D_{1}^{-1}, J E_{1} J=E_{2}^{-1}, J E_{2} J=$ $E_{1}^{-1}, J E_{3} J=E_{2} E_{4}^{-1} E_{5}^{-1} E_{2}^{-1}, J E_{4} J=E_{2} E_{5} E_{3}^{-1} E_{1}^{-1}, J E_{5} J=$ $E_{1} E_{5}^{-1} E_{2}^{-1}$;

(b) $W D_{1} W^{-1}=D_{3} D_{5}^{-1}, W D_{2} W^{-1}=D_{1}^{-1}, W D_{3} W^{-1}=D_{4}^{-1} D_{5}^{-1}$, $W D_{4} W^{-1}=D_{5} D_{1}^{-1}, W D_{5} W^{-1}=D_{2} D_{4}^{-1} D_{5}^{-1}, W E_{1} W^{-1}=$ $E_{3} E_{5}^{-1}, W E_{2} W^{-1}=E_{1}^{-1}, W E_{3} W^{-1}=E_{4}^{-1} E_{5}^{-1}, W E_{4} W^{-1}=$ $E_{5} E_{1}^{-1}, W E_{5} W^{-1}=E_{2} E_{4}^{-1} E_{5}^{-1}$

(c) $T D_{1} T=D_{2}, T D_{2} T=D_{1}, T D_{3} T=D_{5} D_{4}, T D_{4} T=D_{4}^{-1}$, $T D_{5} T=D_{3} D_{4}, T E_{1} T=E_{2}, T E_{2} T=E_{1}, T E_{3} T=E_{5} E_{4}$, $T E_{4} T=E_{4}^{-1}, T E_{5} T=E_{3} E_{4}$;

(d) $\sigma D_{j} \sigma=D_{j}, \sigma E_{j} \sigma=E_{j}, j=1, \ldots, 5$,

we obtain faithful symplectic representations

$$
\left.\rho: \widehat{K} / G_{5} \rightarrow \operatorname{Sp} \widetilde{(10} ; \mathcal{Z}\right)
$$

and

$$
\left.\eta: \widehat{K} / F_{5} \rightarrow \operatorname{Sp} \widetilde{(10} ; \mathcal{Z}\right)
$$


defined by

$$
\rho(\sigma)=\left[\begin{array}{rrrrrrrrrr}
-1 & 0 & 0 & 0 & 0 & 0 & -1 & -1 & 0 & 0 \\
0 & -1 & 0 & 0 & 0 & -1 & 0 & 0 & 0 & -1 \\
0 & 0 & -1 & 0 & 0 & -1 & 0 & 0 & 0 & -1 \\
0 & 0 & 0 & -1 & 0 & 0 & 0 & 0 & 0 & 0 \\
0 & 0 & 0 & 0 & -1 & 0 & -1 & -1 & 0 & 0 \\
0 & 0 & 0 & 0 & 0 & 1 & 0 & 0 & 0 & 0 \\
0 & 0 & 0 & 0 & 0 & 0 & 1 & 0 & 0 & 0 \\
0 & 0 & 0 & 0 & 0 & 0 & 0 & 1 & 0 & 0 \\
0 & 0 & 0 & 0 & 0 & 0 & 0 & 0 & 1 & 0 \\
0 & 0 & 0 & 0 & 0 & 0 & 0 & 0 & 0 & 1
\end{array}\right]
$$




$$
\rho(J)=\left[\begin{array}{rrrrrrrrrr}
-1 & 0 & 0 & 0 & 0 & j_{11} & j_{12} & j_{13} & j_{14} & j_{15} \\
0 & -1 & 0 & 0 & 0 & j_{21} & j_{22} & j_{23} & j_{24} & j_{25} \\
0 & 0 & -1 & 0 & 0 & j_{31} & j_{32} & j_{33} & j_{34} & j_{35} \\
1 & -1 & 1 & 0 & -1 & j_{41} & j_{42} & j_{43} & j_{44} & j_{45} \\
-1 & 1 & -1 & -1 & 0 & j_{51} & j_{52} & j_{53} & j_{54} & j_{55} \\
0 & 0 & 0 & 0 & 0 & -1 & 0 & 0 & 1 & -1 \\
0 & 0 & 0 & 0 & 0 & 0 & -1 & 0 & -1 & 1 \\
0 & 0 & 0 & 0 & 0 & 0 & 0 & -1 & 1 & -1 \\
0 & 0 & 0 & 0 & 0 & 0 & 0 & 0 & 0 & -1 \\
0 & 0 & 0 & 0 & 0 & 0 & 0 & 0 & -1 & 0
\end{array}\right]
$$

In the next three subsections we proceed to show the following results: 
Theorem 8.2. Let $S$ be a maximal symmetric Riemann surface of genus 5 admitting a maximal reflection $\tau: S \rightarrow S$ so that $S / \tau$ is a genus one surface with four holes. Let $p \in(2-\sqrt{3}, 1)$ be so that $S=\Omega / G_{5}$. Then a Riemann matrix of $S$ is given by

$$
\begin{aligned}
& Z=\frac{1}{2}\left[\begin{array}{lllll}
0 & 1 & 1 & 0 & 0 \\
1 & 0 & 0 & 0 & 1 \\
1 & 0 & 0 & 0 & 1 \\
0 & 0 & 0 & 0 & 0 \\
0 & 1 & 1 & 0 & 0
\end{array}\right] \\
& +i\left[\begin{array}{ccccc}
2 u+v & u+v & u+v & v & 2(u+v) \\
u+v & 2 u+v & 2 u+3 v & -v & u+2 v \\
u+v & 2 u+3 v & 2 u+v & v & u \\
v & -v & v & -3 v & 2 v \\
2(u+v) & u+2 v & u & 2 v & 2 u
\end{array}\right], \\
& \text { where }\left\{\begin{array}{l}
u=\frac{-1}{2 \pi} \log \left(\frac{\sqrt{\prod_{\gamma \in G_{2}} \frac{\gamma\left(q_{2}\right)-A_{1}^{-1}(\infty)}{\gamma\left(q_{1}\right)-A_{1}^{-2}(\infty)}}}{\prod_{\gamma \in G_{5}} \frac{\gamma\left(q_{2}\right)-A_{1}^{-2}(\infty)}{\gamma\left(A_{1}^{-1}\left(q_{1}\right)\right)-A_{1}^{-2}(\infty)}}\right) \\
v=\frac{w}{2}-u
\end{array}\right.
\end{aligned}
$$

Theorem 8.3. Let $S$ be a maximal symmetric Riemann surface of genus 5 admitting a maximal reflection $\tau: S \rightarrow S$ so that $S / \tau$ is a genus zero surface with six holes. Let $p \in(2-\sqrt{3}, 1)$ be so that $S=\Omega / F_{5}$. Then a Riemann matrix of $S$ is given by

$$
Z=i\left[\begin{array}{ccccc}
2 u+v & u+v & u+v & v & 2(u+v) \\
u+v & 2 u+v & 2 u+3 v & -v & u+2 v \\
u+v & 2 u+3 v & 2 u+v & v & u \\
v & -v & v & -3 v & 2 v \\
2(u+v) & u+2 v & u & 2 v & 2 u
\end{array}\right]
$$




$$
\text { where }\left\{\begin{aligned}
u & =\frac{-1}{2 \pi} \log \left(\frac{\sqrt{\prod_{\gamma \in F_{2}} \frac{\gamma\left(p_{2}\right)-B_{1}^{-1}(\infty)}{\gamma\left(q_{1}\right)-B_{1}^{-2}(\infty)}}}{\prod_{\gamma \in F_{5}} \frac{\gamma\left(p_{2}\right)-B_{1}^{-2}(\infty)}{\gamma\left(B_{1}^{-1}\left(q_{1}\right)\right)-B_{1}^{-2}(\infty)}}\right) \\
v & =\frac{w}{2}-u
\end{aligned}\right.
$$

\subsection{The case $G_{5}$}

The Riemann matrix $Z=X+i Y \in \mathcal{H}_{5}$ of $S_{5}$ defined by the above symplectic basis is a fixed point of the symplectic group $\rho\left(\widehat{K} / G_{5}\right)$. It follows that: $t_{n m}=j_{n m}=0, w_{11}=w_{15}=-w_{21}=-w_{22}=-w_{31}=$ $-w_{32}=w_{41}=w_{45}=1, w_{22}=w_{23}=w_{24}=w_{31}=w_{24}=w_{25}=w_{31}=$ $w_{34}=w_{35}=w_{41}=w_{42}=w_{43}=w_{44}=w_{45}=w_{52}=w_{53}=w_{54}=0$, and

$$
\begin{gathered}
Z=\frac{-1}{2}\left[\begin{array}{ccccc}
0 & 1 & 1 & 0 & 0 \\
1 & 0 & 0 & 0 & 1 \\
1 & 0 & 0 & 0 & 1 \\
0 & 0 & 0 & 0 & 0 \\
0 & 1 & 1 & 0 & 0
\end{array}\right] \\
+\mathrm{i}\left[\begin{array}{ccccc}
2 u+v & u+v & u+v & v & 2(u+v) \\
u+v & 2 u+v & 2 u+3 v & -v & u+2 v \\
u+v & 2 u+3 v & 2 u+v & v & u \\
v & -v & v & -3 v & 2 v \\
2(u+v) & u+2 v & u & 2 v & 2 u
\end{array}\right],
\end{gathered}
$$

where $0<-v<u$.

\subsection{The case $F_{5}$}

The Riemann matrix $Z=X+i Y \in \mathcal{H}_{5}$ of $R_{5}$ defined by the above symplectic basis is a fixed point of the symplectic group $\eta\left(\widehat{K} / F_{5}\right)$. It 
follows that: $t_{n m}=j_{n m}=w_{n m}=0$, and

$$
Z=i\left[\begin{array}{ccccc}
2 u+v & u+v & u+v & v & 2(u+v) \\
u+v & 2 u+v & 2 u+3 v & -v & u+2 v \\
u+v & 2 u+3 v & 2 u+v & v & u \\
v & -v & v & -3 v & 2 v \\
2(u+v) & u+2 v & u & 2 v & 2 u
\end{array}\right]
$$

where $0<-v<u$.

\subsection{The relations between $w, u$ and $v$}

Let us now consider the regular (unbranched) covering $P: S_{5} \rightarrow S_{2}$ (respectively, $P: R_{5} \rightarrow R_{2}$ ), whose covering group is the Klein group $\mathcal{Z}_{2}+\mathcal{Z}_{2}$ generated by the automorphisms of $S_{5}$ (respectively, $R_{5}$ ) defined by $A_{1}$ and $A_{2}$ (respectively, $B_{1}$ and $B_{2}$ ). We have that $H_{1}(P): H_{1}\left(S_{5}, \mathcal{Z}\right) \rightarrow H_{1}\left(S_{2}, \mathcal{Z}\right)$ (respectively, $H_{1}(P): H_{1}\left(R_{5}, \mathcal{Z}\right) \rightarrow$ $\left.H_{1}\left(R_{2}, \mathcal{Z}\right)\right)$ is given by $H_{1}(P)\left(\theta_{1}\right)=H_{1}(P)\left(\theta_{4}\right)=H_{1}(P)\left(\theta_{5}\right)=\alpha_{1}$, $H_{1}(P)\left(\theta_{2}\right)=H_{1}(P)\left(\theta_{3}\right)=\alpha_{2}, H_{1}(P)\left(\delta_{1}\right)=H_{1}(P)\left(\delta_{5}\right)=2 \beta_{1}$, $H_{1}(P)\left(\delta_{2}\right)=H_{1}(P)\left(\delta_{3}\right)=2 \beta_{2}$ and $H_{1}(P)\left(\delta_{4}\right)=0$.

Let us denote by $w_{1}$ and $w_{2}$ the dual holomorphic one-forms of $\alpha_{1}$ and $\alpha_{2}$, respectively, for the surface $S_{2}$ (respectively, $R_{2}$ ). Similarly, let us denote by $\eta_{1}, \eta_{2}, \eta_{3}, \eta_{4}$ and $\eta_{5}$ the dual holomorphic oneforms of $\theta_{1}, \theta_{2}, \theta_{3}, \theta_{3}$ and $\theta_{5}$, respectively, for the surface $S_{5}$ (respectively, $R_{5}$ ). It follows then the pull-back of holomorphic forms $P^{*}: H^{1,0}\left(S_{2}\right) \rightarrow H^{1,0}\left(S_{5}\right)$ (respectively, $P^{*}: H^{1,0}\left(R_{2}\right) \rightarrow H^{1,0}\left(R_{5}\right)$ ) is defined by $P^{*}\left(w_{1}\right)=\eta_{1}+\eta_{4}+\eta_{5}$ and $P^{*}\left(w_{2}\right)=\eta_{2}+\eta_{3}$. Using the equality

$$
4(u+v) i=\int_{\delta_{1}} \eta_{1}+\eta_{4}+\eta_{5}=\int_{\delta_{1}} P^{*} w_{1}=2 \int_{\beta_{1}} w_{1}=2 i w,
$$

we obtain that $w=2(u+v)$. Again using the results due to Burnside [2] described at the end of section 4 to the groups $G_{5}$ and $F_{5}$ we obtain

$$
2 u+v=-i \int_{\delta_{1}} \eta_{1}=\frac{-1}{2 \pi} \log \left(\prod_{\gamma \in G_{5}} \frac{\gamma\left(q_{2}\right)-A_{1}^{-2}(\infty)}{\gamma\left(A_{1}^{-1}\left(q_{1}\right)\right)-A_{1}^{-2}(\infty)}\right), \quad \text { for } G_{5}
$$


$2 u+v=-i \int_{\delta_{1}} \eta_{1}=\frac{-1}{2 \pi} \log \left(\prod_{\gamma \in F_{5}} \frac{\gamma\left(q_{2}\right)-B_{1}^{-2}(\infty)}{\gamma\left(B_{1}^{-1}\left(q_{1}\right)\right)-B_{1}^{-2}(\infty)}\right), \quad$ for $F_{5}$

From the above two equalities, we obtain the forms for $u$ and $v$ as desired.

\section{References}

[1] Bers, L.: Automorphic forms for Schottky groups. Adv. in Math. 16 (1975), 332-361.

[2] Burnside, W.: On a class of Automorphic Functions. Proc. London Math. Soc. Vol 23 (1892), 49-88.

[3] Conway, J. H. and Sloan, N. J. A.: it Sphere Packings, Lattices and Groups. Springer-Verlag, 1988.

[4] Costa, A. F. and Hidalgo, R. A.: Anticonformal automorphisms and Schottky coverings. Preprint.

[5] Chuckrow, V.: On Schottky groups with application to Kleinian groups. Ann. of Math. 88 (1968), 47-61.

[6] González-Díez, G.: Loci of curves which are prime Galois coverings of $\mathcal{P}_{1}$. Proc. London Math. Soc. 62 (1991), 469-489.

[7] Heltai, B.: Symmetric Riemann surfaces, torsion subgroups and Schottky coverings. Proc. Amer. Math. Soc. 100 (1987), 675-682.

[8] Hidalgo, R. A.: On Schottky groups with automorphisms. Ann. Acad. Scie. Fenn. Ser. AI Mathematica 19 (1994), 259-289.

[9] Hidalgo, R. A.: Schottky uniformizations of closed Riemann surfaces with Abelian groups of conformal automorphisms. Glasgow Math. J. 36 (1994), 17-32. 
[10] Hidalgo, R. A.: Dihedral groups are of Schottky type. Revista Proyecciones. 18 (1999), 23-48.

[11] Hidalgo, R. A.: $\mathcal{A}_{4}, \mathcal{A}_{5}$ and $\mathcal{S}_{4}$ of Schottky type. Preprint.

[12] Hidalgo, R. A.: Bounds for Conformal Automorphisms of Riemann Surfaces with Condition (A). Preprint.

[13] Keen, L.: On hyperelliptic Schottky groups. Ann. Acad. Sci. Fenn. Series A.I. Mathematica 5 (1980).

[14] Marden, A.:Schottky groups and circles. Contribution to Analysis, A collection of papers Dedicated to Lipman Bers. (1994), $273-278$.

[15] Maskit, B.: Kleinian Groups. G.M.W. 287, Springer-Verlag, 1988.

[16] Maskit, B.: Special uniformizations of symmetric Riemann surfaces. Preprint

[17] Maskit, B.: A characterization of Schottky groups. J. d'Analyse Math. 19 (1967), 227-230.

[18] May, C. L.: Automorphisms of compact Klein surfaces with boundary. Pacific J. Math. 59 (1975), 199-210.

[19] May, C. L.: A bound for the number of automorphisms of a compact Klein surface with boundary. Proc. Amer. Math. Soc. 63 (1977), 273-280.

[20] Mumford, D.: Tata lectures on Theta II. Progress on Mathematics 43, Birkhaüser, Boston, 1984.

[21] Natanzon, S. M.: Differential equations for Prym theta-functions, a criterion for two-dimensional finite zone potential Schrödinger operators to be real. Funktsional Anal. i Prilozhen 26 (1992), $17-26$.

[22] Quine, J. R. and Zhang, P.: Extremal symplectic lattices. Preprint 
[23] Schmutz, P.: Riemann surfaces with shortest geodesic of maximal lenght. Gemetric and Functional Analysis, 3 (1993), 564-631.

[24] Sibner, R. J.: Uniformization of Symmetric Riemann surfaces by Schottky groups. Trans. Amer. Math. Soc. 116 (1965), 79-85.

Received : November 2000.

Rubén A. Hidalgo

Departamento de Matemática

Universidad Técnica Federico Santa María

Valparaíso

Chile

e-mail : rhidalgo@mat.utfsm.cl 
